\title{
6 Ausblick
}

Mit dem neuen Brennverlaufsmodell wurde nach Kenntnis des Autors erstmals in der vorliegenden Modellklasse der Verbrennungsfortschritt in der Betriebsart Benzinselbstzündung durch eine Kombination der beiden Mechanismen „Flammenausbreitung“" und „Volumenreaktion“ beschrieben. Dies ermöglicht nicht nur ein besseres Verständnis der in bestimmten Betriebszuständen maßgeblichen Vorgänge, sondern auch einen kontinuierlichen Übergang zum konventionellen fremdgezündeten Betrieb, womit das Modell auch den für die Praxis bedeutsamen Betriebsartenwechsel erfassen kann.

Diese Fähigkeiten des Modells in Kombination mit weiteren vorteilhaften Eigenschaften - der Beschränkung auf wenige, intuitiv verständliche Abstimmparameter und das Erreichen einer sehr geringen Rechenzeit - machen es in hohem Maße dafür geeignet, im täglichen Gebrauch in der virtuellen Motorentwicklung eingesetzt zu werden. Damit bietet es das Potential, den Bedarf an Prüfstandsversuchen zu reduzieren und so der Weiterentwicklung des Brennverfahrens neue Impulse zu geben. Die frühzeitige Motorauslegung, der Aufbau von Betriebs- und Regelstrategien, die Festlegung von Anforderungen an einen variablen Ventiltrieb und allgemein die Untersuchung transienter Vorgänge könnten Aufgaben sein, bei denen das neue Modell ein hilfreiches Werkzeug darstellt.

Daneben bietet das Modell aufgrund seiner prinzipiellen Konzeption mit der gleichzeitigen Erfassung von Flammenausbreitungs- und Selbstzündmechanismen auch aus Modellierungssicht Entwicklungsperspektiven hin zu einem integralen Modell der ottomotorischen Verbrennung, das neben der konventionellen, fremdgezündeten ottomotorischen Verbrennung und der kontrollierten Benzinselbstzündung auch Phänomene wie Klopfen und Vorentflammungen beschreiben kann. Wenngleich auf dem Weg dorthin noch einiges an Arbeit liegt, ist letztlich daraus ein weiter vertieftes Verständnis und ein weiterer Gewinn in der Vorhersagegüte von Modellen zur Beschreibung ottomotorischer Verbrennungsvorgänge zu erwarten. 